\title{
Clinical and socio-demographic characteristics of minors victim of sexual assault
}

\author{
R. Ben Soussia1, S. Khouadja1, W. Bouali', M. Zemzem², I. Marrag ${ }^{1}$, S. Bouslah², L. Zarrouk¹, L. Gaha² \\ 1 University Hospital Mahdia, Tunisia, Department of psychiatry \\ 2 University Hospital Monastir, Tunisia, Department of psychiatry
}

\section{Background and aims}

* Our society has been transformed in terms of communication, which would increase the risk of children's exposure to sexuality. $>$ The aim of our study was to describe the socio-demographic and clinical profile of sexual assault victims.

\section{Materials and Methods}

* A retrospective descriptive study including all children seen at the child psychiatric consult at the University Hospital in Monastir over a 12-year period and who have been suspected of or identified as being sexually assaulted.

- The psychiatric disorders were defined according to the diagnosis criteria of the fifth version of DSM.

\section{Results}

* We have included 93 children and teenagers.

* Average age of $\mathbf{1 0}$ years $\pm \mathbf{3 . 9}$.

* Sex ratio (M/F): 0.97.

* The majority of the study population came from an urban background (100\%) and a nuclear family (75\%).

* $32 \%$ of the cases have an average socio-economic level.

$\$ 84.7 \%$ of the children were schooled.

* Family history of psychiatric and criminal disorders: $48.4 \%$

* Personal medical history: $60.2 \%$

* History of domestic abuse prior to the sexual assault: $31.6 \%$.

* The revelation of the sexual assault was in $44 \%$ of cases in the course of a forensic examination and in $47 \%$ of cases in the course of a child psychiatric consult.

* Sexual assaults were mainly sexual touching (47\%) and vaginal and/or anal penetrations (44\%) (Figure).

* More than three-quarters of assaults (79\%) were committed in a familiar place for the child.

* The assault was unique (47\%) or repetitive (44\%).

* It was associated with another type of violence (26\%).

* Sexual assault was committed by someone known to the child $(54 \%)$, particularly by a family member $(28 \%)$.

* The aggressor was a male (93\%), mainly adult or teenager and there were many of them in $17.6 \%$ of cases.

* The initial psychiatric assessment showed mental disorders in $70 \%$ of cases: depressive disorder and coping disorder with rates of $32 \%$ and $21 \%$ respectively.

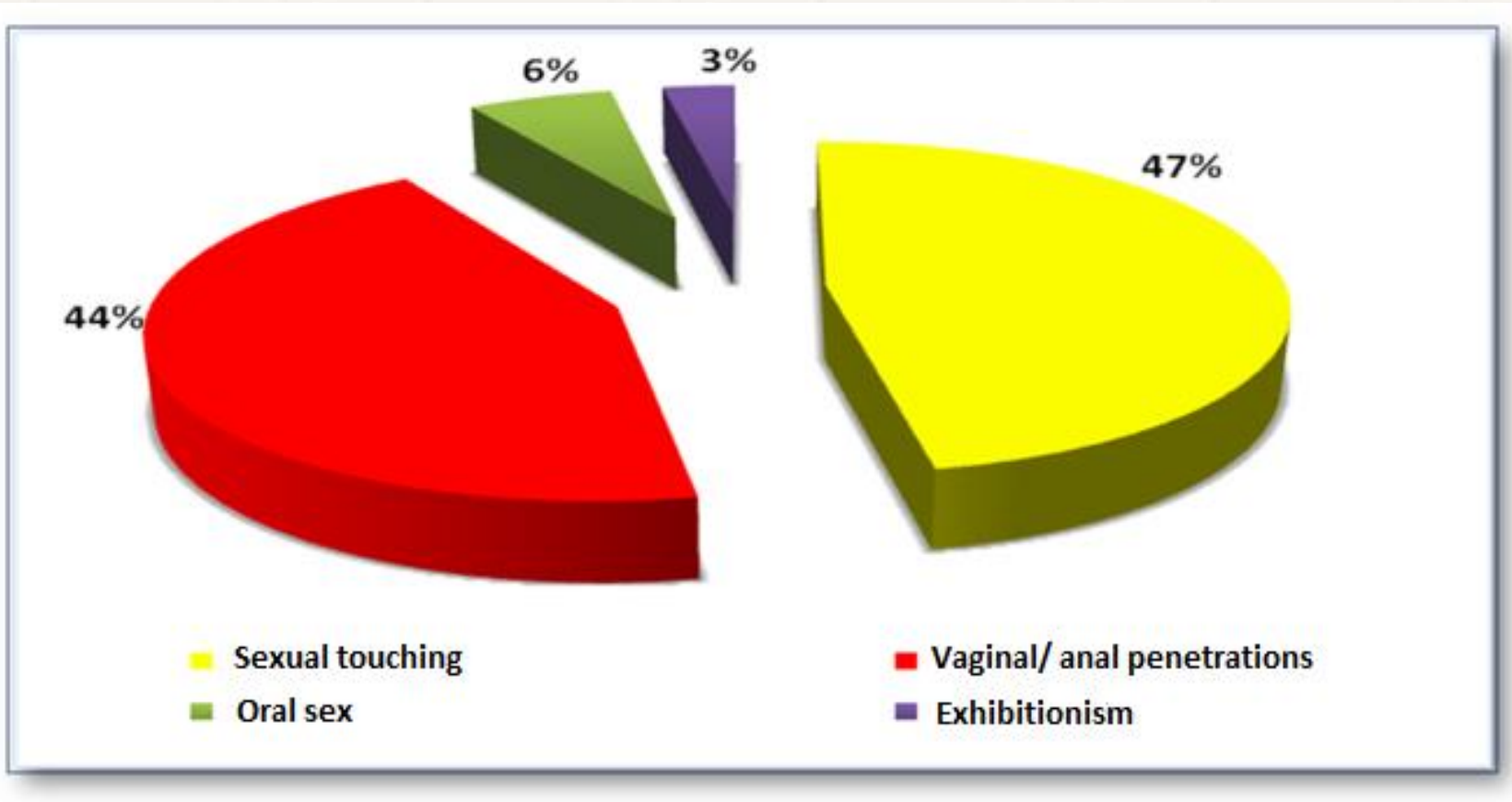

Figure: Type of sexual assault.

\section{Discussion}

* Many studies reported average ages ranging from 10 to 12 years of age at which the majority of victims experience sexual assault $[1,2]$.

- With a peak at 6 years old and a second peak at $13-14$ years old [3]. 10 to $30 \%$ of girls and 10 to $15 \%$ of boys are reported to be victims of sexual assault [3].

- At the national level, a study of 28 expert reports on children examined for sexual abuse found a sex ratio of 1 and an average age of the victim of 8.7 years [4].

* Finkelhor, in a meta-analysis conducted in 1994, noted that the incidence of sexual abuse among children was 7 to $36 \%$ among girls and 3 to $29 \%$ among boys.

* Indeed, several studies have shown that girls are 1.5 to 3 times more likely to be sexually abused than boys [5].

- An unpleasant socio-economic, relational and emotional family environment is a factor of vulnerability according to most studies [6,7].

* According to a general population survey conducted by Bagley et al in 1985, touching and cuddling accounted for $45 \%$ followed by penetration or attempting to penetrate in $15 \%$ [8]. - $85 \%$ of the aggressors are known by the victim and $3 / 4$ of the abuses are committed by the family environment [4].

\section{Conclusion}

* The outcomes of sexual abuse of minors are a real public health problem.

* The severity of the consequences of this violence on the victims' mental and physical health is further heightened by being locked in the trap of silence about the events they endured. 